\title{
Probing the Epoch of Reionization with Low Frequency Arrays
}

\author{
S. Zaroubi
}

\author{
Kapteyn Astronomical Institute, University of Groningen, Landleven 12, 9747AD Groningen, The Netherlands
}

\begin{abstract}
The Epoch of Reionization (EoR) is the epoch in which hydrogen in the Universe reionize after the "Dark Ages". This is the second of two major phase transitions that hydrogen in the Universe underwent, the first phase being the recombination era in which hydrogen became neutral at redshift $\approx 1100$. The EoR, occurs around $z \approx 10$ and is probably caused by the first radiation emitting astrophysical sources, hence it is crucial to our understanding of when and how the Universe "decided" to start forming astrophysical objects and how that influenced subsequent structure formation in the Universe. As such, the EoR is related to many fundamental questions in cosmology, galaxy formation, quasars and very metal poor stars; all are foremost research issues in modern astrophysics. The redshifted $21 \mathrm{~cm}$ hyperfine line is widely considered as the most promising probe for studying the EoR in detail. In the near future a number of low frequency radio telescopes (LOFAR, MWA, GMRT and SKA) will be able to observe the $21 \mathrm{~cm}$ radiation arriving from the high redshift Universe. In this paper I present our current picture of the ionization process, review the $21 \mathrm{~cm}$ line physics and discuss the challenges that the current generation experiments are expected to face. Finally, I discuss the potential of SKA in exploring the EoR and the Universe's Dark Ages.
\end{abstract}

\section{Introduction}

The last couple of decades have witnessed the emergence of an overarching paradigm, the $\Lambda \mathrm{CDM}$ model, that describes the formation and evolution of the Universe and its structure. The $\Lambda$ CDM model accounts very successfully for most of the available observational evidence on large scales. According to this paradigm at redshift of 1100 , about 400,000 years after the Big Bang, the Universe's temperature and density decreased enough to allow the ions and electrons to recombine into neutral hydrogen and helium (and a very small percentage of heavier elements). Immediately afterwords, photons decoupled from the baryonic material and the Universe became transparent leaving a relic radiation, known as the cosmic microwave background $(\mathrm{CMB})$ radiation. To date, the $\mathrm{CMB}$ radiation has been observed in many experiments providing one of the most compelling evidences for the CDM paradigm (for recent results see the WMAP papers, e.g., Spergel et al. 2007; Page et al. 2007; Komatsu et al. 2010).

The matter-radiation decoupling has ushered the Universe into a period of darkness as its temperature dropped below $3000 \mathrm{~K}$ and steadily decreased with the Universe's expansion. These Dark Ages ended about 400 million years later, when the first radiation emitting objects (stars, black-holes, etc.) were formed and assembled into protogalaxies. The first objects then began to produce a spectrum of radiation, especially ionizing ultraviolet photons. After a sufficient number of ionizing sources had formed, the temperature and the ionized fraction of the gas in the Universe increased rapidly and most of the neutral hydrogen eventually ionized. This period, during which the cosmic gas went from being almost completely neutral to almost completely ionized, is known as the Epoch of Reionization of the Universe.

The most accepted picture on how reionization unfolds is simple. The first radiation-emitting objects ionize their immediate surroundings, forming ionized bubbles that expand until the neutral intergalactic medium (hereafter, IGM) consumes all ionizing photons. As the number of objects increases, so do the number and size of the bubbles, until they eventually fill the whole Universe. However, most of the details of this scenario are yet to be clarified. For example: what controls the formation of the first objects and how much ionizing radiation do they produce? How do the bubbles expand into the intergalactic medium and what do they ionize first, high-density or low density regions? The answer to these questions and many others that arise in the context of studying the EoR touches upon many fundamental questions in cosmology, galaxy formation, quasars and very metal poor stars; all are foremost research issues in modern astrophysics. A substantial theoretical effort is currently dedicated to understanding the physical processes that trigger this epoch, govern its evolution, and the ramifications it had on subsequent structure formation (c.f., Barkana \& Loeb 2001; Bromm \& Larson 2004; Ciardi \& Ferrara 2005; Choudhury \& Ferrara 2006; Furlanetto \& Briggs 2006). However, and despite the pivotal role played by the EoR in cosmic history, observational evidence on it is very scarce and, when available, is indirect and model dependent.

It is generally acknowledged that the $21 \mathrm{~cm}$ emission line from neutral hydrogen at high redshifts is the most promising probe for studying the Dark Ages and the EoR in detail. HI fills the IGM except in regions surrounding the ionizing radiation of the first objects to condense out of the cosmic flow. Computer simulations suggest that we may expect an evolving complex patch work of neutral (HI) and ionized hydrogen (HII) regions (Gnedin \& Abel 2001; Ciardi et al. 2001; Ritzerveld et al. 2003; Susa 2006; Razoumov \& Cardall 2005; Nakamoto et al. 2001; Whalen \& Norman 2006; Rijkhorst et al. 2005; Mellema et al. 2006; Zahn et al. 2007; Mesinger \& Furlanetto 2007; Pawlik \& Schaye 2008; Thomas et al. 2009). The current constraint strongly suggest that the EoR roughly straddles the redshift range of $z \sim 20-6$. 
In this contribution I will give a short overview of the present picture of the Universe's reionization and review the potential of current and future instruments -focusing mostly on LOFAR and SKA- in detecting it. The paper is organized as follows. In section 2 a review of the current observational constraint is given. In section 3 the possible reionization sources are discussed. Section 4 presents the physics of the $21 \mathrm{~cm}$ line with the factors that determine the spin and brightness temperature (the observable in these experiments) and show a number of results obtained from numerical simulations. In $\S 5$ the expected foregrounds, the instrument sensitivity and the predicted power spectrum measurement and the skewness are discussed. The paper ends with conclusions.

\section{Current observational constraints}

Currently, there are a few strong observational constraints on the EoR. The CMB temperature and polarization data obtained by the WMAP satellite allow measurement of the total Thomson scattering of the primordial CMB photons off intervening free electrons produced by the EoR along the line of sight (Page et al. 2007; Spergel et al. 2007; Komatsu et al. 2010). They show that the CMB radiation has, on its way to us, only been damped by $\sim 9 \%$, indicating that the Universe was mostly neutral for 400 million years and then ionized. However, the Thomson scattering measurement is an integral constraint telling us little about the sources of reionization, its duration or how it propagated to fill the whole Universe.

The second strong constraint comes from specific features in the spectra of distant quasars, known as the Lyman-alpha forest. These features, which are due to neutral hydrogen, indicate two important facts about reionization: 1) hydrogen in the recent Universe is highly ionized with neutral fraction of $\sim 10^{-4}$; 2) at redshift 6.5 , i.e., about 900 million years after the Big Bang, the neutral fraction of hydrogen suddenly increases demarcating the end of the reionization process (Fan et al. 2003, 2006). Despite these data providing strong constraints on the ionization state of the Universe at redshifts below 6.5, they say very little about the reionization process itself (although see Bolton \& Haehnelt 2007).

A third, albeit weaker, constraint comes from measuring the temperature of the intergalactic medium at the redshift range 4-6. The widths of the hydrogen Lyman- $\alpha$ absorption lines can be used to determine the IGM temperature. This property has been used by a number of authors who have measured the IGM temperature at $\mathrm{z} \approx 4$ (Schaye et al. 2000; Theuns et al. 2002). The temperature of the diffuse IGM at these redshifts depends on its reionization history because the thermal timescales are long. Theuns et al. (2002) and Hui \& Haiman (2003), have used these measurement at redshift about 4 to extrapolate back in time where they argue that, for a single-phase reionization scenarios, hydrogen reionization occurred below redshift $\mathrm{z}=9$. Recently, Bolton et al. (2010) have measured the IGM temperature at even higher redshift $(\mathrm{z} \approx 6)$ and employed similar arguments to conclude that reionization happened at redshift lower than 9. As said, this constraint is somewhat weaker than the previous two as it requires assumptions about the heating



Fig. 1: This figure shows a sketch of the likely development of the EoR. About 500,000 years after the Big Bang $(z \sim 1000)$ hydrogen recombined and remained neutral for a few hundred million years (the Dark Ages). At a redshift, $z \sim 15$, the first stars, galaxies and quasars began to form, heating and reionizing the hydrogen gas. The neutral IGM can be observed with LOFAR through its redshifted $21 \mathrm{~cm}$ spinflip transition up to redshift 11.5. However, many atmospheric, galactic and extra-galactic contaminants corrupt the $21 \mathrm{~cm}$ signal.

and cooling rates from the redshift at which the temperature is measured all the way back to the reionization redshift.

A whole slew of possible other constraints currently discussed in the literature are either very controversial, very weak or, as is often the case, both. Most are very interesting and exciting; but can only be investigated reliably with a new generation of instruments that are expected to come online within a decade.

To summarize, from the current observational constraints and recent numerical and theoretical models of structure formation a very simple picture of the Universe's reionization history has emerged. A cartoon of the various phases and the objects featuring in this simple picture is shown in Figure 1.

\section{Astrophysical Ionization Sources}

The nature of the first ionizing sources is unclear. In general two types of astrophysical sources are considered ${ }^{\text {a }}$ Population III stars or miniquasars, both of which represent hypothetical but plausible populations of the first objects in the universe, and that are significant sources of ionizing photons.

First stars: The impact of stellar sources have been studied by many authors (e.g., Cen 2003; Ciardi et al. 2003; Haiman \& Holder 2003; Sokasian et al. 2003; Wyithe \& Loeb 2003). These studies find in general that in order to provide enough ionizing flux at or before $z=15$, for the usual scale-invariant primordial density fluctuation power spectrum,

\footnotetext{
a Other ionization sources, e.g., decaying dark matter particles, are possible but less likely
} 
one needs Population III stars, which provide about 20 times more ionizing photons per baryon than Population II (Schaerer 2002; Bromm et al. 2001), or an IMF that initially is dominated by high mass stars. This is in agreement with recent numerical simulations of the formation of first stars from primordial molecular clouds that suggest that the first metal-free stars were predominantly very massive, $m_{*} \lesssim 100 M_{\odot}$ (Abel et al. 2000; Bromm et al. 2002).

Mini-quasars: Miniquasars have also been considered as a significant ionising source (e.g., Kuhlen \& Madau 2005; Oh 2001; Madau et al. 2004; Ricotti, \& Ostriker 2004a,b; Zaroubi $\&$ Silk 2005; Zaroubi et al. 2007). In view of the correlation between central black hole mass and spheroid velocity dispersion (Ferrarese 2002; Gebhardt et al. 2000), this correlation demonstrates that seed black holes must have been present before spheroid formation. Theory and observations suggests that the seeds from which the super massive black holes amounted to at least $1000 \mathrm{M}_{\odot}$ and were in place before $z \sim 10$ (Fan et al. 2003, 2006; Madau \& Rees 2001; Silk \& Rees 1998).

Decaying Dark Matter particles: In the literature, the influence of dark matter particles annihilation or decay on reionization is considered. The consensus here is that, given the constraints that the CMB impose on the value of Thomson optical depth, these process are unlikely to substantially contribute to the Universe's ionization. Instead many authors consider whether the influence of annihilation/decay of dark matter particles on reionization could be used, despite its small amplitude, to constraint the properties of these particles.

\section{The $21 \mathrm{~cm}$ Spectral Line as a Probe of the EoR}

In recent years it has become clear that the $21 \mathrm{~cm}$ line can be used to probe the neutral IGM prior to and during reionization. This hyperfine transition line of atomic hydrogen (in the ground state) arises due to interactions between and the electron and proton spins (Hogan \& Rees 1979; Scott \& Rees 1990; Madau et al. 1997). The excited triplet state is a state in which the spins are parallel whereas the spins at the lower (singlet) state are antiparallel. The $21 \mathrm{~cm}$ line is a forbidden line with a probability of $2.9 \times 10^{-15} \mathrm{~s}^{-1}$ corresponding to a life time of about $10^{7}$ years. Despite its low decay rate the $21 \mathrm{~cm}$ transition line is one of the most important astrophysical probes, simply due to the vast amounts of hydrogen in the Universe (Ewen, \& Purcell 1951; van de Hulst 1945; Muller \& Oort 1951)

\subsection{The $21 \mathrm{~cm}$ Spin Temperature}

The intensity of the $21 \mathrm{~cm}$ radiation is controlled by one parameter, the so called spin temperature, $T_{\text {spin }}$. This temperature is defined through the equation, $n_{1} / n_{0}=3 \exp \left(-T_{*} / T_{\text {spin }}\right)$, where $n_{1}$ and $n_{0}$ are the number densities of electrons in the triplet and singlet states of the hyperfine levels, and $T_{*}=0.0681 \mathrm{~K}$ is the temperature corresponding to the $21 \mathrm{~cm}$ wavelength.

In his seminal papers George Field (Field 1958, 1959) used the quasi-static approximation to calculate the spin temperature, $T_{\text {spin }}$, as a weighted average of the CMB temperature, $T_{C M B}$, the gas kinetic temperature, $T_{k i n}$, and the temperature related to the existence of ambient Lyman- $\alpha$ photons, $T_{\alpha}$ (Wouthuysen 1952; Field 1959). For almost all interesting cases one can safely assume that $T_{k i n}=T_{\alpha}$ (Field 1958; Furlanetto \& Briggs 2006; Madau et al. 1997). The spin temperature is given by:

$$
T_{\text {spin }}=\frac{T_{C M B}+y_{k i n} T_{k i n}+y_{\alpha} T_{k i n}}{1+y_{k i n}+y_{\alpha}},
$$

where $y_{\text {kin }}$ and $y_{\alpha}$ are the kinetic and Lyman- $\alpha$ coupling terms, respectively. The kinetic coupling term is due to collisional excitations of the $21 \mathrm{~cm}$ transitions. The Lyman- $\alpha$ coupling term is due to the so called Lyman- $\alpha$ pumping mechanism - also known as the Wouthyusen-Field effect - which is produced by photo-exciting the hydrogen atoms to their Lyman transitions (Field 1958, 1959; Wouthuysen 1952). Finally, it is important to note that for the $21 \mathrm{~cm}$ radiation to be observed it has to attain a different temperature than that of the CMB background (Field 1958, 1959; Hogan \& Rees 1979; Wouthuysen 1952).

Since the decoupling mechanisms can influence the spin tem perature differently, it is important to explore the decoupling issue for various types of ionization sources. For instance, stars decouple the spin temperature mainly through radiative Lyman- $\alpha$ pumping whereas mini-quasars decouple it through a combination of collisional Lyman $-\alpha$ pumping and heating (Zaroubi et al. 2007; Chuzhoy et al. 2006), both produced by energetic secondary electrons ejected due to the miniqso x-ray photons (Shull \& van Steenberg 1985). The difference between the spin temperature decoupling patterns between the two will eventually help disentangle the nature of the first ionization sources.

Figure 2 shows the expected global evolution of the spin temperature as a function of redshift. The blue solid line represents $T_{C M B}$, which drops as $1+z$. The green line shows the gas temperature as a function of redshift. At $z \gtrsim 200$ the gas temperature is still coupled to the CMB due to Compton scattering off residual electrons leftover from the recombination era. At redshift $\sim 200$, however, the gas decouples from the CMB and starts adiabatically cooling as a function of $(1+z)^{2}$ until the first objects start forming and heating up the gas at redshift below 30. The spin temperature (shown by the red lines) has a somewhat more complicated behavior, at $z>30$ it is coupled to the gas temperature due to collisional coupling caused by a residual electrons leftover from recombination. At $z \approx 100$ the efficiency of collisional coupling to the gas drops due to the Hubble expansion. At this stage, the spin temperature starts veering towards $T_{C M B}$ until it is completely dominated by it. At lower redshifts the first astrophysical objects that heat and ionize the IGM couple $T_{\text {spin }}$ to the gas. Here, broadly speaking, there are two possible histories, one in which $T_{\text {spin }}$ couples to the gas as it heats up where it obtained temperature greater than $T_{C M B}$ (red solid line). In the other possible evolution the spin temperature couples to the gas much before the kinetic temperature exceeds that of the CMB (red dashed line) (Thomas et al. 2010; Baek et al. 2009). In the former case the $21 \mathrm{~cm}$ radiation, after decoupling from the CMB at $z \lesssim 30$, is seen only in emission, whereas in the latter case it is seen initially in absorption and only at later stages in emission. 




Fig. 2: The global evolution of the CMB (blue line), gas (green line) and spin (red solid line and red dashed line) temperatures as a function of redshift. The CMB temperature evolves steadily as $1+\mathrm{z}$ whereas the gas and spin temperatures evolve in a more complicated manner (see text for detail).

\subsection{The Brightness Temperature}

In radio astronomy, where the Rayleigh-Jeans law is usually applicable, the radiation intensity, $I(v)$ is expressed in terms of the brightness temperature, so that

$$
I(v)=\frac{2 v^{2}}{c^{2}} k_{B} T_{b},
$$

where $v$ is the radiation frequency, $c$ is the speed of light and $k_{B}$ is Boltzmann's constant (Rybicki, \& Lightman 1986). This in turn can only be detected differentially as a deviation from $T_{C M B}$. The predicted differential brightness temperature deviation from the cosmic microwave background radiation is given by Field (1958, 1959); Ciardi \& Madau (2003),

$$
\begin{aligned}
\delta T_{b}=28 \mathrm{mK}(1+\delta) & x_{H I}\left(1-\frac{T_{C M B}}{T_{\text {spin }}}\right) \times \\
& \times\left(\frac{\Omega_{b} h^{2}}{0.0223}\right) \sqrt{\left(\frac{1+z}{10}\right)\left(\frac{0.24}{\Omega_{m}}\right),}
\end{aligned}
$$

where $h$ is the Hubble constant in units of $100 \mathrm{~km} \mathrm{~s}^{-1} \mathrm{Mpc}^{-1}, \delta$ is the mass density contrast, $x_{H I}$ is the neutral fraction, and $\Omega_{m}$ and $\Omega_{b}$ are the mass and baryon densities in units of the critical density. Note that the three quantities, $\delta, x_{H I}$ and $T_{\text {spin }}$, are all functions of 3D position.

One also should add to this equation the contribution of redshift distortion. In the linear regime of gravitational instability this component is simple to add (Kaiser 1987). However, in the quasi-linear regime this term is not well understood and should be explored in more detail.

Equation 3 gives the cosmological signal we are after and clearly shows the complication and, at the same time, the wealth of information that could be extracted from the EoR signal. For example, according to recent simulations (e.g., Iliev et al. 2008; Thomas et al. 2009) the ionized fraction of the IGM at $z \approx 11$ is very small. If the spin temperature is much larger than $T_{C M B}$ the brightness temperature is proportional to the cosmological density and can provide a clean probe of the primordial density field. However, at later stages one can probe the evolution of the ionized fraction as a function of redshift which provides information about the ionization sources and their clustering properties and so on. Figure 3 shows a typical distribution of the differential brightness temperature as predicted by the recent Thomas et al. (2009) simulations.

One should also note that in most simulations the spin temperature is assumed to be much larger than the CMB temperature, namely term $\left(1-T_{C M B} / T_{\text {spin }}\right)$ in eq. 3 is 1 . As figure 2 shows, this is a good assumption at the later stages of reionization, however, it is not valid at the early stages. Modeling this effect is somewhat complex and requires radiative transfer codes that capture the Lyman- $\alpha$ line formation and multifrequency effects, specially those coming from energetic photons (Baek et al. 2009; Thomas et al. 2010).

\section{The redshifted $21 \mathrm{~cm}$ Observation}

The current generation of radio telescopes $\left(\mathrm{LOFAR}^{\mathrm{b}}, \mathrm{MWA}^{\mathrm{c}}\right.$ GMRT, and $21 \mathrm{CMA}^{\mathrm{d}}$ ) will capture the lower redshift part of the $\delta T_{b}$ evolution $(z \lesssim 12)$. LOFAR, for example have two observational bands, high band and low band. The high band array is expected to be sensitive enough for measuring the redshifted $21 \mathrm{~cm}$ radiation coming from the neutral IGM within the redshift range of $\mathrm{z}=11.4(115 \mathrm{MHz})$ to $\mathrm{z}=6(203 \mathrm{MHz})$, with a resolution of 3-4 arcminutes and a typical field of view of $\sim 120$ square degrees (with 5 beams) and a sensitivity of the order of $80 \mathrm{mK}$ per resolution element and $1 \mathrm{MHz}$ frequency bandwidth. At frequencies below the FM band, probed by the low band array, the LOFAR sensitivity drops significantly and the sky noise increase dramatically that detection of HI signals at these frequencies is beyond reach with LOFAR (Harker et al. 2010; Jelić et al. 2008; Labropoulos et al. 2009). The main and most challenging task of the LOFAR EoR project is to extract the cosmological signal from the data and interpret it.

In the future SKA can significantly improve on the current instruments in two major ways. Firstly, it will have at least an order of magnitude higher signal-to-noise which will allow much better statistical detection of the EoR and give us access to the Universe's Dark Ages which corresponds to much higher redshifts $(z \approx 35)$ hence providing crucial information about cosmology which non of the current telescope hopes to have. Secondly, SKA will have a resolution better by a factor of few at least relative to the current telescopes. These two advantages will not only improve on the understanding we gain with current telescopes but give the opportunity to address a host of fundamental issues that current telescope will not be able to address at all. Here I give a few examples: 1- Due to the limited resolution and poor signal-to-noise, the nature of the ionizing sources is expected to remain poorly constrained; 2- the

\footnotetext{
b http://www.lofar.org \& http://www.astro.rug.nl/ LofarEoR

c http://www .mwatelescope.org

${ }^{d}$ http://21cma.bao.ac.cn
} 




Fig. 3: Position-redshift slices through the image-frequency volume of the reionization simulations of Thomas et al. (2009).

mixing between the astrophysical effects and the cosmological evolution is severe during the EoR but much less so during the Dark Ages an epoch beyond the reach of the current generation of telescope but within SKA reach; 3- at redshifts larger than 30 the $21 \mathrm{~cm}$ could potentially provide very strong constraints, much more so than the $\mathrm{CMB}$, on the primordial non-gaussianity of the cosmological density field which is essential in order to distinguish between theories of the very early Universe (e.g., distinguish between different inflationary models).

In section 4 we discussed the cosmological $21 \mathrm{~cm}$ signal and showed that it is expected to be of the order of $\approx 10 \mathrm{mK}$, However, the detectable signal in the frequency range that corresponds the epoch of reionization is composed of a number of components each with its own physical origin and statistical properties. These components are : 1 - the $21 \mathrm{~cm}$ signal coming from the high redshift Universe. 2- galactic and extra-galactic foregrounds, 3- ionospheric influences, 4- telescope response effects and 5- noise (see Figure 1). Obviously, the challenge of the upcoming experiments is to distill the cosmological signal out of this complicated mixture of influences. This will crucially depend on the ability to calibrate the data very accurately so as to correct for the ever changing ionospheric effects and variation of the instruments response with time. In this section I present the various non cosmological components and briefly discuss their properties.

\subsection{Station configuration and uv coverage}

The low frequency arrays must be configured so that they have a very good uv-coverage. This is crucial to the calibration effort of the data where a filled uv plane is important for obtaining precise Local (Nijboer et al. 2006) and Global (Smirnov \& Noordam 2004) Sky models (LSM/GSM; i.e. catalogues of the brightest, mostly compact, sources in and outside of the beam, i.e. local versus global). It is also crucial for the ability to accurately fit for the foregrounds (Harker et al. 2009b; Jelić et al. 2008) and to the measurement of the EoR signal power spectrum (Bowman et al. 2006; Harker et al. 2010; Hobson \& Maisinger 2002; Santos et al. 2005)

The uv coverage of an interferometric array depends on the layout of the stations (interferometric elements), their number and size as well as on the integration time, especially, when the number of stations is not large enough to have a good instantaneous coverage.

For a given total collecting area one can achieve a better uv coverage by having smaller elements (stations). For example LOFAR has chosen to have large stations resulting in about $\approx 10^{3}$ baselines in the core area. Such a small number of baselines needs about 5-6 hours of integration time per field in order to fill the uv plane (using the Earth's rotation). In comparison, MWA which has roughly $1 / 2$ the total collecting area of LOFAR chose to have smaller stations with about $\approx 10^{5}$ baselines resulting in an almost instantaneous full uv coverage.

The decision on which strategy to follow has to do with a number of considerations that include the ability to store the raw visibilities, hence, allowing for a better calibration, an acceptable noise level for both the foreground extraction needs as well the power spectrum measurement (see the following sections; $\S 5.2$ and $\S 5.3$ ). A compromise between these issues as well the use of the telescopes for other projects is what drives the final lay out of the antennas.

\subsection{Noise Issues}

The thermal noise level for a given visibility, i.e., uv point, is,

$$
\Delta V(u, v) \approx \frac{2 k_{B} T_{s y s}}{\epsilon d A \sqrt{B t}},
$$

where $T_{s y s}$ is the system temperature, $\epsilon$ is the efficiency, $d A$ is the station area, $B$ is the bandwidth and $t$ is the observation time (see e.g., Morales 2005). This expression is simple to 
understand, the more one observes, either in terms of integration time, frequency band width or station collecting area the less uncertainty one has. Obviously, if the signal we are after is well localized in either time, space or frequency the relevant noise calculation should take that into account.

In order to calculate the noise in the $3 \mathrm{D}$ power spectrum, the main quantity we are after, one should remember that the frequency direction in the observed datacube is proportional to the redshift which in turn can be easily translated to distance whereas the $u$ and $v$ coordinates are in effect Fourier space coordinates. Therefore, to calculate the power spectrum first first shoud Fourier transform the data cube along the frequency direction. Following Morales (2005) I will call the new Fourier space coordinate $\eta$ (with $d \eta$ resolution) which together with $\mathrm{u}$ and $\mathrm{v}$ define the Fourier space vector $\boldsymbol{u}=\{u, v, \eta\}$. From this, one can calculate the noise contribution to the power spectrum at a given $|\boldsymbol{u}|$,

$$
P_{\text {noise }}(|\mathbf{u}|) \approx 2 N_{\text {beam }}^{-1} N_{\text {cell }}^{-1 / 2}\left(\frac{2 k_{B} T_{\text {sys }}}{\epsilon d A d \eta}\right)^{2} \frac{1}{B n(|\boldsymbol{u}|) t},
$$

where $N_{\text {beam }}$ is the number of simultaneous beams that could be measured, $N_{\text {cell }}$ is the number of independent Fourier samplings per annulus and $n(|\boldsymbol{u}|)$ is the number of baselines covering this annulus (Morales 2005). Note that $n(|\boldsymbol{u}|)$ is proportional to square number of stations, hence, $n(|\boldsymbol{u}|) d A^{2}$ is proportional to the square of the total collecting area of the array regardless of the station size. This means that the noise power spectrum measurement does not depend only on the total collecting area, band width and integration time, it also depends the number of stations per annulus. This is easy to understand as follows, the power in a certain Fourier space annulus is given by the variance of the measured visibilities in the annulus which carries uncertainty proportional to the inverse square root of number of points. This point is demonstrated in figure 4. As an example, MWA will have the advantage of having many samplings of the uv plane (100 times more than LOFAR) whereas LOFAR will have the advantage of simultaneous multi-beams and somewhat larger collecting area.

\subsection{The Foregrounds}

The foregrounds in the frequency regime $(40-200 \mathrm{MHz})$ are very bright and dominate the sky. In fact the amplitude of the foreground contribution, $T_{s k y}$, at $150 \mathrm{MHz}$ is about 4 orders of magnitude larger than that of the expected signal. However, since we are considering radio interferometers the important part of the foregrounds is that of the fluctuations which reduces the ratio between the them and the cosmological signal to about 2-3 orders of magnitude, which is still a formidable obstacle to surmount.

The most prominent foreground is the synchrotron emission from relativistic electrons in the Galaxy, this source of contamination contributes about $75 \%$ of the foregrounds. Other sources that contribute to the foreground are radio galaxies, galaxy clusters, resolved supernovae remnant, free-free emission provide $25 \%$ of the foreground contribution (see Shaver

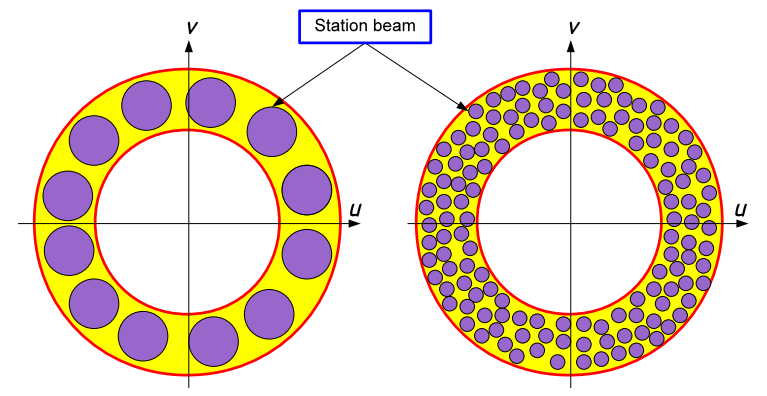

Fig. 4: This figure shows how two different experiments might sample an annulus in uv. The size of uv point is given by the station (interferometric element) size, larger station (left panel) has a larger footprint relative to the smaller station case (right panel) in uv plane; the footprint is shown by the purple circles. Even though the sampled area in the two cases might be the same, the fact that smaller stations sample the annulus more results in an increased accuracy in their estimation of the power spectrum.


Fig. 5: A figure showing the various cosmological and galactic components that contribute to the measured signal at a given frequency, The slices are color coded with different tales owing to the vast difference between the range of brightness temperature in each component, however the figure shows the rms of the galactic foregrounds, extra galactic foregrounds and cosmological signal

et al. 1999). Figure 5 shows simulated foreground contribution at $120 \mathrm{MHz}$ taken into account all the foreground sources mentioned.

As many studies have shown, the very smooth structure of the foreground sources along the frequency direction will enable disentangling their contribution from that of the cosmological signal. The foregrounds are normally fitted by some procedure (e.g., polynomial fitting (Jelić et al. 2008), or more advanced non parametric methods (Harker et al. 2009b)) in order to recover the EoR cosmological signal; Figure 6 shows how successful such a recovery is. 




Fig. 6: Statistical detection of the EoR signal from the LOFAR-EoR data maps that include diffuse components of the foregrounds and realistic instrumental noise $\left(\sigma_{\text {noise }}(150 \mathrm{MHz})=52 \mathrm{mK}\right)$. Black dashed line represents standard deviation $(\sigma)$ of the noise as a function of frequency, cyan dashed line $\sigma$ of the residuals after taking out smooth foregrounds component and red solid line the $\sigma$ of original EoR signal. The grey shaded surface represents the $90 \%$ of detected EoR signals from 1000 independent realisations of the noise, where cyan dashed line is mean of detected EoR signal. Note that the y-axis is in logarithmic scale (Jelić et al. 2008).

\section{The Statistics of the observed cosmological signal}

\subsection{The $21 \mathrm{~cm}$ Power Spectrum}

One of the main aims of the EoR projects is to measure the power spectrum of variations in the intensity of redshifted 21$\mathrm{cm}$ radiation from the EoR. As shown in Equation 3 the power spectrum depends on a number of astrophysical and cosmological quantities. The sensitivity with which this power spectrum can be estimated depends on the level of thermal noise (Eq. 5) and sample variance, and also on the systematic errors arising from the extraction process, in particular from the subtraction of foreground contamination. In the LOFAR case, for example, we model the extraction process using realistic simulations of the cosmological signal, the foregrounds and noise. In doing so we estimate the sensitivity of the LOFAR EoR experiment to the redshifted $21 \mathrm{~cm}$ power spectrum. Detection of emission from the EoR should be possible within 360 hours of observation with a single station beam. Integrating for longer, and synthesizing multiple station beams within the primary (tile) beam, then enables us to extract progressively more accurate estimates of the power at a greater range of scales and redshifts (see Figure 7 Harker et al. 2010).



Fig. 7: Power spectra of the cosmic signal (blue solid line), the noise (red dashed line), the residuals (magenta dotted line) and the extracted signal (black points with error bars) at three different redshifts.for the case when the uv coverage is frequency-dependent, we have 300 hours of observation per frequency channel with a single station beam, and the foreground fitting is done using Wp smoothing in Fourier space (Harker et al. 2010).

\subsection{High order statistics: Skewness and Kurtosis}

Figure 8 showes the PDF of the brightness temperature at four different redshifts; the PDF is clearly nongaussian at all four cases. High order moments, like the skewness, as a function of redshift could be a useful tool for signal extraction in the presence of realistic overall levels of foregrounds and noise. Harker et al. (2009a) have shown that the cosmological signal, under generic assumptions, have a very characteristic pattern in the skewness as a function of redshift (Figure 9). At sufficiently high redshifts the signal is controlled by the cosmological density fluctuations which, in the linear regime, are Gaussian. At lower redshifts, and as nonlinearity kicks in, the signal starts getting a slightly positive skewness. As the ionization bubbles begin to show up the skewness starts veering towards 0 until it crosses it to the negative side when the weight of the ionized bubbles becomes more important than the high density outliers -note high density outliers are likely to ionize first- but the distribution is still dominated by the density fluctuations. At lower redshift the bubbles dominate the PDF and the neutral areas become the outliers giving rise to a sharp positive peak to the skewness. At redshift around 6 the instrument noise assumed to be Gaussian dominates driving the skewness again towards zero. Exploiting this characteristic behavior might allow us to pick up the cosmological signal with this high order statistic. 




Fig. 8: The distribution of $\delta T_{\mathrm{b}}$ in a certain cosmological simulation of reionization (Iliev et al. 2008) at four different redshifts, showing how the PDF evolves as reionization proceeds. Note that the y-axis scale in the top two panels is different from that in the bottom two panels. The delta-function at $\delta T_{\mathrm{b}}=0$ grows throughout this period while the rest of the distribution retains a similar shape. The bar for the first bin in the bottom-right panel has been cut off: approximately 58 per cent of points are in the first bin at $z=7.78$ (Harker et al. 2009a).



Fig. 9: Skewness of the fitting residuals from data cubes with uncorrelated noise, but in which the residual image has been denoised by smoothing at each frequency before calculating the skewness. The three lines correspond to results from three different simulations (Thomas et al. 2009; Iliev et al. 2008). Each line has been smoothed with a moving average (boxcar) filter with a span of nine points. The grey, shaded area shows the errors, estimated using 100 realizations of the noise (see Harker et al. 2009a)

\section{Conclusions}

The imminent availability of observations of redshifted $21 \mathrm{~cm}$ radiation from the Universe's Dark Ages and the EoR will be one of the most exciting development in the study of cosmology and galaxy and structure formation in recent years. Currently, there are a number of instruments that are designed to measure this radiation. In this contribution I have argued that despite the many difficulties that face such measurements they will provide a major breakthrough in our understanding of this crucial epoch. In particular current radio telescopes, such as LOFAR, will be able to provide us with the global history of the EoR progression, the fluctuations power spectrum during the EoR, etc., up to $z \approx 11$. These measurements will usher the study of the high redshift Universe into a new era which will bridge, at least in part, the large gap that currently exists in observation between the very high redshift Universe $(z \approx 1100)$ as probed by the CMB and low redshift Universe $(z \lesssim 6)$.

Although the current generation of telescopes have a great promise they will also have limitations. For example they have neither the resolution, the sensitivity nor the frequency coverage to address many fundamental issues, like the nature of the first sources. Crucially, they will not provide a lot of informa tion about the Dark Ages which is only accessible through very low frequencies in the range of $40-120(z \approx 35-11)$.

Fortunately, in the future SKA can improve dramatically on the current instruments in three major ways. Firstly, it will have at least an order of magnitude higher signal-to-noise which will allow much better statistical detection of the EoR. Secondly, it will give us access to the Universe's Dark Ages which corresponds to much higher redshifts $(z \sim 30)$ hence providing a truly pristine probe of cosmology. Thirdly, SKA will have, at least, a factor of few better resolution in comparison with the current telescopes, thus better constraining the dominant ionization sources. In summary these three advantages will not only improve on the understanding we gain with current telescopes but give the opportunity to address a host of fundamental issues that current telescope will not be able to address.

The next decade will be extremely exciting for studying the high redshift Universe, especially as these radio telescope gradually come online, starting with LOFAR and MWA. As they promise to resolve many of the puzzles we have today pertaining to the formation and evolution of the first object, cosmology and the physical process in the high redshift intergalactic medium.

Acknowledgements. Many of the results shown here have been obtained in collaboration with the members of the LOFAR EoR project whose contribution I would like to acknowledge.

\section{References}

Abel T., Bryan G. L., Norman M. L., 2000, ApJ, 540, 39.

Baek S., Di Matteo P., Semelin B., Combes F., Revaz Y., 2009, A\&A, 495, 389

Barkana R., Loeb A., 2001, PhR, 349, 125

Bolton J. S., Becker G. D., Wyithe J. S. B., Haehnelt M. G., Sargent W. L. W., 2010, arXiv, arXiv:1001.3415 
Bolton J. S., Haehnelt M. G., 2007, MNRAS, 382, 325

Bowman J. D., Morales M. F., Hewitt J. N., 2006, ApJ, 638, 20

Bromm, V., Coppi, P.S., Larson, R.B., 2002, ApJ, 564, 23.

Bromm V., Kudritzki R. P., Loeb A., 2001, ApJ, 552, 464

Bromm V., Larson R. B., 2004, ARA\&A, 42, 79

Cen R., 2003, ApJ, 591, L5

Choudhury T. R., Ferrara A., 2006, arXiv:astro-ph/0603149

Chuzhoy L., Alvarez M. A., Shapiro P. R., 2006, ApJ, 648, L1

Ciardi B., Ferrara A., Marri S., Raimondo G., 2001, MNRAS, 324, 381

Ciardi B., Madau P., 2003, ApJ, 596, 1

Ciardi B., Ferrara A., White S. D. M., 2003, MNRAS, 344, L7

Ciardi B., Ferrara A., 2005, SSRv, 116, 625

Ewen, H.I., Purcell, E.M., 1951, Nature 168: 356-357

Fan X., et al., 2003, AJ, 125, 1649

Fan X., et al., 2006, AJ, 131, 1203

Ferrarese L., 2002, ApJ, 578, 90

Field, G.B., 1958, Proc, IRE, 46, 240.

Field, G.B., 1959, ApJ, 129, 551.

Furlanetto, S., Oh, P., Briggs, F., 2006, Physics Reports

Gebhardt K., et al., 2000, ApJ, 539, L13

Gnedin N. Y., Abel T., 2001, NewA, 6, 437

Haiman Z., Holder G. P., 2003, ApJ, 595, 1

Harker G. J. A., et al., 2009a, MNRAS, 393, 1449

Harker G. J. A., et al., 2009b, MNRAS, 397, 1138

Harker G. J. A., et al., 2010, MNRAS, submitted.

Hobson M. P., Maisinger K., 2002, MNRAS, 334, 569

Hogan C. J., Rees M. J., 1979, MNRAS, 188, 791

Hui L., Haiman Z., 2003, ApJ, 596, 9

van de Hulst, H.C., 1945, Ned. Tijdschr. Natuuurk. 11: 210-221

Iliev I. T., Mellema G., Pen U.-L., Bond J. R., Shapiro P. R., 2008, MNRAS, 384, 863

Jelić V., et al., 2008, MNRAS, 389, 1319

Kaiser N., 1987, MNRAS, 227, 1

Kuhlen M., Madau P., 2005, MNRAS, 363, 1069

Komatsu E., et al., 2010, arXiv:1001.4538

Labropoulos P., et al., 2009, arXiv:0901.3359

Madau P., Meiksin A., Rees M. J., 1997, ApJ, 475, 429

Madau P., Rees M. J., 2001, ApJ, 551, L27

Madau P., Rees M. J., Volonteri M., Haardt F., Oh S. P., 2004, ApJ, 604, 4

Mellema G., Iliev I. T., Alvarez M. A., Shapiro P. R., 2006, NewA, 11, 374

Mesinger A., Furlanetto S., 2007, ApJ, 669, 663

Morales, M.F., 2005, ApJ, 619,678

Muller, C.A., Oort, J.H., 1951, Nature 168: 357-358

Nakamoto T., Umemura M., Susa H., 2001, MNRAS, 321, 593

Nijboer R. J., Noordam J. E., Yatawatta S. B., 2006, ASPC, 351,291

Oh S. P., 2001, ApJ, 553, 499

Page L., et al., 2007, ApJS, 170, 335 (astro-ph/0603450)

Pawlik A. H., Schaye J., 2008, arXiv, 802, arXiv:0802.1715

Razoumov A. O., Cardall C. Y., 2005, MNRAS, 362, 1413

Ricotti, M., Ostriker, J. P., 2004a, MNRAS, 350, 539.

Ricotti, M., Ostriker, J. P., 2004b, MNRAS, 352, 547

Rijkhorst E.-J., Mellema G., Icke V., 2005, A\&A, 444, 849

Ritzerveld J., Icke V., Rijkhorst E.-J., 2003, astro, arXiv:astro$\mathrm{ph} / 0312301$
Rybicki, G. B., \& Lightman, A. P. 1986, Radiative Processes in Astrophysics, by George B. Rybicki, Alan P. Lightman, ISBN 0-471-82759-2. Wiley-VCH , June 1986.,

Santos M. G., Cooray A., Knox L., 2005, ApJ, 625, 575

Schaye J., Theuns T., Rauch M., Efstathiou G., Sargent W. L. W., 2000, MNRAS, 318, 817

Scott D., Rees M. J., 1990, MNRAS, 247, 510

Schaerer D., 2002, A\&A, 382, 28

Shaver, P. A., Windhorst, R. A., Madau, P., \& de Bruyn, A. G. 1999, A \& A, 345, 380

Shull J.M., van Steenberg M.E., 1985, ApJ, 298, 268

Silk J., Rees M.J., 1998, A\&A, 331, L1

Smirnov O. M., Noordam J. E., 2004, ASPC, 314, 18

Sokasian A., Abel T., Hernquist L., Springel V., 2003 MNRAS, 344, 607

Spergel D. N., et al., 2007, ApJS, 170, 377 (astro-ph/0603449)

Susa H., 2006, PASJ, 58, 445

Theuns T., Schaye J., Zaroubi S., Kim T.-S., Tzanavaris P., Carswell B., 2002, ApJ, 567, L103

Thomas R. M., et al., 2009, MNRAS, 393, 32

Thomas R. M., Zaroubi S., 2010, MNRAS, submitted

Whalen D., Norman M. L., 2006, ApJS, 162, 281

Wouthuysen S. A., 1952, AJ, 57, 31

Wyithe J. S. B., Loeb A., 2003, ApJ, 588, L69

Zahn O., Lidz A., McQuinn M., Dutta S., Hernquist L.T Zaldarriaga M., Furlanetto S. R., 2007, ApJ, 654, 12

Zaroubi S., Silk J., 2005, MNRAS, 360, L64

Zaroubi S., Thomas, R.M., Sugiyama, N., Silk J., 2007, MNRAS, 375, 1269 